\title{
Uncertainty Propagation for Energy Dispersive X-ray Spectrometry
}

\author{
Nicholas W. M. Ritchie ${ }^{1}$, Dale E. Newbury ${ }^{1}$ \\ ${ }^{1}$ National Institute of Standards and Technology, Gaithersburg, MD 20899-8371
}

Most of us learned the rules for basic uncertainty propagation in university. These rules allow us to estimate how uncertainty in an input parameter is transformed into an equivalent uncertainty in an output or measured parameter. These rules are based on a first-order Taylor expansion of the transformation about the value of the input parameter. There are also rules for calculating the combined uncertainty when there are multiple independent or correlated input parameters. The definitive resource for application of these rules to measurement problems is the Guide to Uncertainty in Measurement[1]. These rules have been applied to basic electron probe microanalysis by Marinenko and Leigh[4].

However, this guide only alludes to the situation in which there are multiple input quantities which result in multiple output quantities - so called multivariate measurement models. Figure 1 shows the difference between the many-to-one and the many-to-many situations. There are two complementary approaches to dealing the the many-to-many situation - the Supplement 2 approach[3] and the Monte Carlo approach[2]. The first approach is the multi-dimensional analog to the famililar Taylor series approach. This approach can be expressed in a deceptively simple-looking expression. If $\mathbf{f}(\mathbf{x})$ is a vector-valued function of a vector argument $\mathbf{x}$ the uncertainty in $\mathbf{f}(\mathbf{x}), \mathbb{U}(\mathbf{f}(\mathbf{x}))$ as a function of the uncertainty in the input parameter, $\mathbf{x}, \mathbb{U}(\mathbf{x})$ is

$$
\mathbb{U}\left(\mathbf{f}\left(\mathbf{x}_{0}\right)\right)=\left.\mathbb{J}_{\mathbf{f}(\mathbf{x})} \mathbb{U}(\mathbf{x}) \mathbb{J}_{\mathbf{f}(\mathbf{x})}{ }^{\top}\right|_{x_{0}}
$$

where $\mathbb{J}_{\mathbf{f}(\mathbf{x})}$ is the Jacobian of $\mathbf{f}(\mathbf{x})$ and $\mathbb{U}(\mathbf{x})$ is the uncertainty or covariance matrix for $\mathbf{x}$. The Jacobian is defined as the matrix containing the partial derivatives of the functions in $f(x)$ with respect to the variables $\mathbf{x}$. The expression is evaluated at $\mathbf{x}_{0}$, the best estimate of the input parameters, $\mathbf{x}$.

The Monte Carlo approach involves evaluating the expression $\mathbf{f}(\mathbf{x})$ for many values of $\mathbf{x}$ drawn from the distribution described by $\mathbb{U}(\mathbf{x}) . \mathbb{U}(\mathbf{f}(\mathbf{x}))$ is then estimated from the distribution of the output values. Both methods should produce similar results in most situations although the Monte Carlo method is more flexible.

X-ray microanalysis involves many expressions of the many-to-many type. For example, the transformation we apply to the measured k-ratios to estimate the composition is many-to-many. It is not possible to estimate the quantity of one element from the k-ratio associated with that element without measuring all the other elements in the material. While we typically write the matrix correction equation[5] in terms of a single element, $A$, as

$$
k_{A}=\frac{C_{A} \cdot Z A F_{u n k}\left(C_{A}, C_{B}, \ldots\right)}{C_{s t d} \cdot Z A F_{s t d}\left(C_{A}, C_{B_{s t d}}, \ldots\right)},
$$

were $Z A F\left(C_{A}, C_{B}, \ldots\right)$ represents either $Z \cdot A \cdot F$-type or or $\phi(\rho z)$-type correction factors, this is really shorthand for a set of coupled equations. There is an additional peculiarity to this expression. The desired value, the composition, is expressed as a function of itself and the measured value, the k-ratio, placing this problem into the class of implicit multivariate measurement models[3], which require slightly more complex methods to address. Furthermore, we'd like to be able to take into estimate the influence of uncertainties in the composition of the standard to the unknown. The composition of the standard comes 

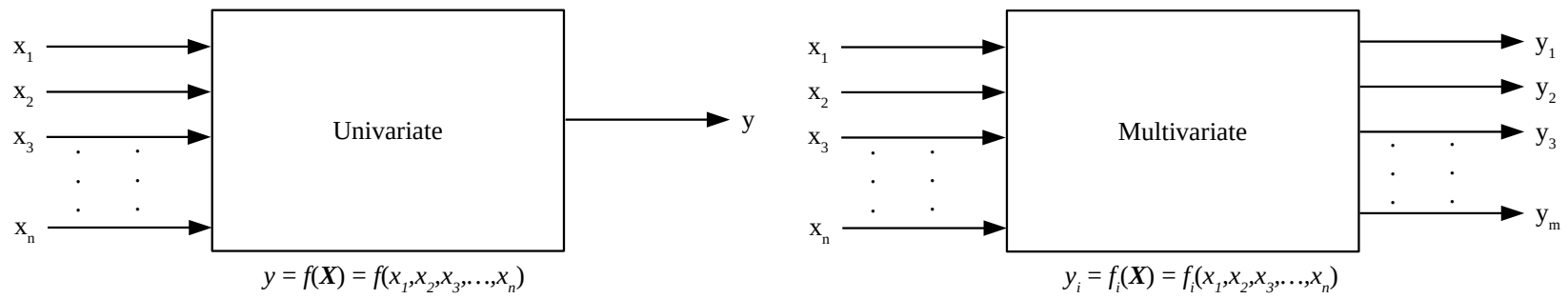

Figure 1. Comparing a univariate measurement model (left) to a multivariate measurement model (right).

into play in a complex manner through the matrix corrections and through "Castaing's first approximation" $\left(k_{A} \approx C_{A} / C_{B}\right)$.

Typically, the k-ratios measured in a wavelength dispersive measurement are largely independent because the depend only upon the number of counts in the peak and associated background measurements which do not interfere. Energy dispersive spectrometer measurements are often not independent because the breadth of the peak plus background is sufficient that the range-of-channels associated with one element interferes with the range-of-channels associated with another. This is reflected in the covariance matrix associated with the output from linear least squares fitting. (There is a secondary related issue associated with the filter often used to mitigate the continuum.)

We will present tools that we have developed for investigating error propagation using both the JCGM 102 ("Jacobian") and the JCGM 101 ("Monte Carlo") approaches. We will demonstrate applications to the domains of spectrum fitting and matrix correction. These insights can allow us to make better measurements by understanding how measurement choices like choice of standard, beam energy, acquisition time and x-ray transition and uncertainties in various input parameters influence the accuracy of our results. Information like this can be used to optimize the measurement parameters we do control and mitigate the influence of parameters we can't control.

References:

[1] Joint Committee for Guides in Metrology (2008). JCGM 100: 2008 - Evaluation of Measurement Data - Guide for the Expression of Uncertainty in Measurement., International Organization for Standardization (ISO) 1-167.

[2] Joint Committee for Guides IN Metrology (2008). JCGM 101:2008 - Evaluation of Measurement DataSupplement 1 to the Guide to the Expression of Uncertainty in Measurement - Propagation of Distributions using a Monte Carlo Method., International Organization for Standardization (ISO) .

[3] Joint Committee For Guides IN Metrology (2011). JCGM 102: 2011 - Evaluation of Measurement Data - Supplement 2 to the "Guide to the Expression of Uncertainty in Measurement" - Extension to Any Number of Output Quantities, International Organization for Standardization (ISO) $1-80$.

[4] Marinenko, R. \& Leigh, S. (2010). Uncertainties in Electron Probe Microanalysis, IOP Conference Series: Materials Science and Engineering, vol. 7, 012017, IOP Publishing.

[5] Pouchou, J. \& Pichoir, F. (1991). Quantitative Analysis of Homogeneous or Stratified Microvolumes Applying the Model 'PAP', K. Heinrich \& D. Newbury (eds.), Electron Probe Quantitation, 31-75, Springer. 